\title{
Reproductive intentions and choices among HIV-infected individuals in Cape Town, South Africa: Lessons for reproductive policy and service provision from a qualitative study
}

Diane Cooper

Hillary J. Bracken

Population Council

Landon Myer

Virginia Zweigenthal

Jane Harries

See next page for additional authors

Follow this and additional works at: https://knowledgecommons.popcouncil.org/departments_sbsr-hiv How does access to this work benefit you? Let us know!

\section{Recommended Citation}

Cooper, Diane, Hillary J. Bracken, Landon Myer, Virginia Zweigenthal, Jane Harries, Phyllis Orner, Nontuthuzelo Manjezi, and Pumeza Ngubane. 2005. "Reproductive intentions and choices among HIVinfected individuals in Cape Town, South Africa: Lessons for reproductive policy and service provision from a qualitative study," Policy brief, September. Cape Town: Women's Health Research Unit, University of Cape Town, and Population Council. 


\section{Authors}

Diane Cooper, Hillary J. Bracken, Landon Myer, Virginia Zweigenthal, Jane Harries, Phyllis Orner, Nontuthuzelo Manjezi, and Pumeza Ngubane 

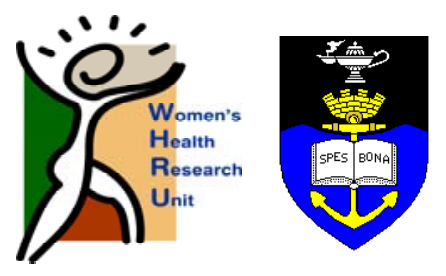

\title{
Policy Brief
}

\section{Reproductive intentions and choices among HI V-infected individuals in Cape Town, South Africa: Lessons for reproductive policy and service provision from a qualitative study}

\author{
Diane Cooper ${ }^{a}$, Hillary Brackenc, Landon Myer ${ }^{b}$, Virginia Zweigenthal ${ }^{\mathrm{a}}$, J ane Harries ${ }^{\mathrm{a}}$, \\ Phyllis Orner ${ }^{a}$, Nontuthuzelo Manjezi ${ }^{a}$, and Pumeza Ngubane ${ }^{a}$
}

\section{BACKGROUND}

While many HIV-infected individuals do not wish to have children, others desire children despite their infected status. The desire and intent to have children among HIV-infected individuals may increase because of improved quality of life and survival following commencement of anti-retroviral treatment. In developing countries such as South Africa, where the largest number of people living with HIV/AIDS worldwide reside, specific government reproductive health policy and service provision for HIV-infected individuals is underdeveloped.

This Brief presents findings from a qualitative study that explored HIV-infected individuals' reproductive intentions, decision-making, and need for reproductive health services. The study also assessed the opinions of health service providers, policymakers, and influential figures within non-governmental organisations (NGOs) who are likely to play important roles in the shaping and delivery of reproductive health services. We focus on issues that impact reproductive choice and decisionmaking and identify critical policy, health service, and research related matters to be addressed.

\section{METHODS}

The study was conducted at two health centres in the Cape Town metropolitan area in South Africa from May 2004 to January 2005. In-depth interviews were conducted with 40 HIV-positive women and 20 HIVpositive men. Half of these respondents ( 20 women and 10 men) had been receiving anti-retroviral treatment (ART) for longer than six months and half were not undergoing ART. Fourteen in-depth interviews were conducted with health care providers delivering HIV care or treatment at the health care facilities where the HIV-positive women and men were interviewed. In addition, 12 interviews were conducted with public sector policymakers and managers and influential figures within HIVIAIDS and reproductive health NGOs. For the HIV-infected respondents, interviews were conducted in their first language, Xhosa. For other respondents interviews were conducted in English. Purposive and snowball sampling techniques were used to select study participants, and data were analysed using a grounded theory approach.

\footnotetext{
${ }^{a}$ Women's Health Research Unit, UCT, ${ }^{b}$ Infectious Diseases Epidemiology Unit, UCT, 'Population Council, New York
} 


\section{KEY FI NDI NGS}

\section{Participant characteristics}

Average time since HIV diagnosis was 2.5 years for women (range 2 weeks- 8 years) and 2.8 years for men (range 2 weeks- 10 years). The average age of female (range 20-45 years) and male (range 26-57 years) respondents was 27 and 37 years respectively. Twenty-seven of the 40 women had an intimate partner, as did 18 of the 20 men. Women had an average of 1.4 children and men an average of 2 . Ten women and 6 men had no live children.

\section{Reproductive intentions}

Not intending to reproduce

I would be committing a great $\sin$ if I would have another child

knowing..this one is sick. I would be putting responsibility on other people $\&$ killing myself.

- woman on ART

\section{Intending to reproduce}

People who are unable to have children find it difficult to lead happy lives.

\section{- man on ART}

Let's say I have no child at all, I would say to the doctor that please I need this child.

- woman not on $A R T$

I have never had a child in my life so I would love to have a child of my own.

$$
\text { - man on } A R T
$$

\section{Social influences}

When I am married I will have to have a baby because...only I and my boyfriend... are aware that I am HIVpositive and... people will ask why am I not becoming pregnant in marriage.

- woman not on ART

There are guys who criticise that you don't have a child yet.

$$
\text { -man on ART }
$$

[The community] question[s] a woman falling pregnant whilst she knew her [HIV] status.

- woman on ART
The 14 health care providers consisted of eight nurse practitioners, three doctors, and three counsellors. All eight of the public sector policymakers and managers were health professionals, whereas the four NGO representatives had varying backgrounds.

\section{Reproductive intentions of $\mathbf{H I} \mathbf{V}$-infected individuals}

Health concerns coloured the reproductive intentions of most of the women and some of the men. Many HIV-infected women and men were firm in their desire not to have children. Both women and men feared infecting a partner or their baby and were anxious about leaving either living or future children as orphans. Women and men were concerned about their ability to financially support their children, given their illness. Women who had given birth to an infected child expressed mixed feelings about becoming pregnant again.

At the same time, in common with uninfected women and men, many HIV-infected individuals felt that children gave meaning to their lives and gave them a reason to live. Many expressed a desire to "leave something of themselves behind" after they died. Children represented a realization of hope or a sign of "normality." Some women expressed a desperate need to have a child, especially if they had no live children. One man reported that his decision to reproduce was shaped by a desire to have a larger family, similar to that of his father and another expressed a desire for a son.

\section{Social influences on reproductive intentions}

Married women, in particular, reported strong family pressure to reproduce, especially if they had not disclosed their HIV status. One woman stated that if she did not bring children into a marriage, her child from a previous relationship would be stigmatised. In other cases, where women had disclosed their status to family, they were discouraged from bearing children. Sometimes this was due to concern for the woman's own health. Women seemed more influenced by family attitudes than men. Some men felt that their masculinity was tied to their ability to reproduce and maintain a family. 
Most HIV-infected women felt community attitudes towards them having children would be negative. However, women and men appeared to be less influenced by the opinions of the community or their friends than those of family. Both women and men were strongly influenced by partners' attitudes towards childbearing. Disclosure was critical in terms of discussion of desires. Women and men on ART were far more likely to have disclosed their status to their partner and to have discussed having children. Some women, especially married women, felt pressured by a partner to have a child against their will; others felt strongly that the decision was theirs and that they would rather leave their partner than be coerced into childbearing. Men tended to report feeling that pregnancy and childbirth was a woman's prerogative and that if their partner wished to have children and they did not, they would submit to their partner's wishes.

\section{Role of peri-natal transmission prevention (PMTCT) programs and ART in shaping reproductive intentions}

Some women felt that the availability of peri-natal transmission prevention (PMTCT) would influence them in favour of having children, sometimes wishing to replace a deceased child or to "do it right" and ensure that the child was not infected. However, a number of women felt that this would not influence them, if their own health remained compromised.

Women and men on ART overwhelmingly experienced positive effects on their health and some felt that being on ART would alter attitudes towards childbearing. Some women on ART, however, feared that their medication could deform their infants in utero. Some men believed the drugs they were on could affect the health of a child they fathered.

\section{Perceptions of health care providers' respect for HIV patients' reproductive choice}

Most HIV-infected women and men had not discussed their fertility desires and intentions with a health care provider because of anticipated negative reactions. Women tended to see doctors and nurses as handling matters related to care and treatment, with the psychosocial aspects being handled by counsellors. Some women who had discussed reproduction with a health care provider found providers to be supportive of their reproductive choice. Others encountered providers who expressed negative attitudes towards HIV-infected women becoming pregnant. In some cases, this was even to the point of providers in ante-natal care settings pressing women to terminate their pregnancy. Women on ART, when disagreeing with providers opinions, tended to stand their ground.

Men, more than women, tended to feel that health care providers would be impartial or sympathetic. Men tended to be more likely to discuss their desires with a doctor than a nurse. Nurses reported that they believed HIV infected men were more concerned with sexual performance than with reproductive intention. 
Client knowledge and experience of services

We just arrive at the [family planning] clinic and provide them with our cards... I don't think they know if we are HIV-positive or negative or what my dates are for the next appointment. - woman on ART

\title{
Clients' knowledge and experience of reproductive health and HIV prevention and treatment services
}

\author{
Contraception and barrier methods of infection prevention
}

Men had little knowledge of services for contraception, emergency contraception, and termination of pregnancy. HIV-infected women used reproductive health services mainly for contraception. Some women were hesitant to use hormonal contraceptives because of perceived side effects or, among women on ART, fear of drug interactions. The quality of contraceptive counseling was variable and there appeared to be little discussion of dual protection and emergency contraception. Some women complained about contraceptive services outside of the HIV care or treatments setting as they felt the range of contraceptive options were limited and there was little information on contraception in the context of HIV. The topic of termination of pregnancy elicited little discussion.

Women and men repeatedly mentioned the importance of using a condom to prevent HIV transmission and re-infection; providers, too, mentioned the great effort they put into promoting condom use. However, individuals found it difficult to reconcile safer sex messages with their desire to reproduce

\section{Prevention of peri-natal transmission of HIV}

Some female respondents had accessed PMTCT services when pregnant. Among women not on ART, knowledge of PMTCT and where to obtain services was poorer. Women perceived PMTCT counselling on infant feeding, contraception, and dual protection to be inadequate. Many women not receiving ART knew that antiretroviral drugs were available at the clinics they were attending and knew that they did not yet qualify for the drugs. They knew little of the health effects of the therapy, however. Their knowledge appeared to be acquired from the community and was limited to vague statements regarding "improved health." Men not on ART displayed only a vague awareness of ART, where it could be obtained, and what benefits it may bring to their lives.

\section{Views and opinions of policymakers, NGO officials, and health service providers}

\section{Views on reproduction}

Many policymakers and health care providers were ambivalent about HIV-infected individuals becoming pregnant. While acknowledging the need for women's reproductive choice, some felt it unwise with respect to public health and of questionable ethics for HIV-infected women to become pregnant. Providers believed they were offering HIV-infected women reproductive choice. Several policymakers and providers recognized, however, that the emphasis with clients tended to be on the consequences of becoming pregnant. Some providers acknowledged that withholding judgment when discussing pregnancy in HIV-positive women was difficult. One nurse described how she sometimes became 'angry' when an HIV-positive woman came for a pregnancy examination and refused to be counselled about

\section{Views on reproduction}

We tend to adopt a medical model and ignore the...social and economic factors that make it difficult... We don't see an HIV positive person as a normal person who has normal needs and wants a family. We don't see them as needing the full scope of advantages that everyone else has.

- public sector health care manager family planning methods. Several policymakers and managers stressed the need to take into account the patient's perspective and provide more sympathetic and effective counselling regarding reproduction.

Providers believed that although counselling should be ongoing, the most crucial moments for counselling were before and after voluntary counselling and testing (VCT) and at onset of ART. Several nurses and counsellors also mentioned the value of couples counselling.

Lack of specific policy or guidelines 
There was no specific policy or set of guidelines for dealing with reproductive choice among HIV-infected individuals. Policymakers noted concern about the lack of guidelines, as well as about insufficient training in contraception, inadequate dual method counselling and the scant reference made to emergency contraception. Providers were unaware of World Health Organization guidelines on contraception for HIVinfected individuals. They expressed concern about the potential for some anti-retroviral (ARV) drugs to cause fetal malformation and felt women on ARVs should use contraception.

Some policymakers spoke of the need for values-clarification training for policymakers and health care providers, as part of ongoing training, to foster greater respect for client's individual wishes and choices.

\section{Problems with integration}

Policymakers, NGO leaders, and public sector service managers remarked that many women only discovered their HIV status upon becoming pregnant. The absence of integration of VCT into routine reproductive health care or other services and of reproductive health care into HIV care and treatment made it difficult to meet HIV-infected individuals' comprehensive health care needs.

\section{CONCLUSION}

The framing of reproductive choice for HIV-infected individuals as either an ethical or medical issue allows for little acknowledgment of the complexities of reproductive decisionmaking. Under circumstances in which many HIV-infected individuals, intentionally or unintentionally, continue to have children, not dealing openly with their fertility desires and intentions makes it difficult to optimally meet their reproductive health care needs. Promoting open discussion is respectful of health clients' rights and encourages safer pregnancy and childbirth through active reproductive planning.

\section{RECOMMENDATIONS}

For many HIV-infected women and men, health care workers can play a central role in decisionmaking about pregnancy. Counselors' roles in discussing reproductive intentions are seen as particularly crucial. Providers need to help manage reproductive health in HIV-infected individuals in a more upfront and structured manner, to probe their reproductive desires and intentions in a less judgmental manner and to support reproductive choice. Providers frequently find themselves working in situations with scarce resources, lack of information and training, and fear of HIV infection. These factors need to be considered in creating a health care environment supportive of the reproductive rights of clients and the needs of heath care workers. The following are key policy, health service, and research recommendations:

\section{POLICY}

- There is a need for specific and overt policy that can help to ensure reproductive choice and improved access to contraception and other reproductive health services for HIV-positive individuals.

\section{HeALTH SERVi Ce}

\section{Training of health care workers}

- Training of health care workers in how to deal sensitively with reproduction in HIV-infected individuals needs to be included in the training curricula of health institutions. Specific information and training is needed on contraception for HIV-infected individuals, on potential interactions between ART and hormonal contraceptives, on the impact of HIV and of ART on pregnancy, on issues related to sexual desire and functioning, and on probing for and dealing with violence against HIV-infected women. 


\section{Counselling}

- Non-judgmental counselling that examines future reproductive desires with HIV-infected clients and planning is needed to assist clients in acquiring knowledge and accessing reproductive health services appropriate to their needs. Clients need information and counselling on contraceptive options and their possible interaction with ART. They need information and open counselling on terminating an unwanted pregnancy. Advice is needed from health care workers on how to balance safer sex practices and the desire to reproduce, including information on the safest ways for HIVinfected individuals to conceive and have a healthy pregnancy.

- More public education is necessary on ART, particularly for HIV-infected individuals not on ART. Information and counselling is also needed on the risks for specific birth defects associated with different ARV drugs.

- $\quad$ HIV care and treatment could provide an important opportunity to provide more information and counselling to men that would strengthen their involvement in reproductive health services more generally.

\section{Health service organization}

- Information on what services exist, what they provide, and where they are located should be compiled to improve knowledge of and access to reproductive services, such as contraception, HIV prevention, STI treatment, cervical screening, PMTCT and obstetric services, as well as HIV care and ART. This information could be disseminated to clients during counselling, care, or treatment sessions.

- Models for better integration of services need to be explored. Further examination is needed of the merits of combining reproductive health, STI services, HIV treatment, and TB and child health services. This might reduce the stigma associated with clinics that previously only provided services for HIV-positive clients, and may allow individuals to make fewer visits to health facilities.

\section{RESEARCH}

- Quantitative research is needed to determine the generalisability of some of the findings of this study.

- Participatory action research is needed among HIV-infected individuals, policymakers, NGO officials and health care providers to discuss and refine the interventions proposed here for improving reproductive health choice and service provision for HIV-infected individuals.

- Model structural interventions need to be tested in service sites to provide evidence that can inform policy and best practice.

- Further qualitative research is needed to explore selected issues such as violence related to reproductive decision-making; experiences of pregnancy termination among HIV-infected women; gaps in reproductive information and service needs for HIV-infected men and ways of addressing these; the impact on HIV-infected women of discovering their HIV status during pregnancy or childbirth; and equitable access to ART treatment and reproductive choice.

\section{Acknowledgements}

The authors would like to thank: T. Mgoqi for the male interviews. N. Nywagi \& Social Trends for transcription. The Western Cape Province and City of Cape Town Health Departments \& managers \& staff at the two research health facilities. Desmond Tutu HIV Centre \& Population Council staff for their insights. Population Council for final editing \& layout. The research was supported by grants from the William \& Flora Hewlett Foundation, the Medical Research Council of South Africa, the Universitv of Cape Town. \& the World Health Oraanisation.
U
Diane Cooper, Women's Health Research Unit
School of Public Health \& Family Medicine
University of Cape Town, Anzio Road, Observatory, 7925, South Africa
Tel: 27214066528 • Fax: 27214488151 or 4066163 • Email: dic@cormack.uct.ac.za • Website: www.whru.uct.ac 